\title{
WP 14_10
}

\author{
George Deltas
}

Department of Economics, University of Illinois, U.-C., United States

\author{
Thanasis Stengos
}

Department of Economics, University of Guelph, Canada

RCEA, Italy

Eleftherios Zacharias

Department of Economics, Athens School of Economics, Greece

\section{Product Line Pricing in a Vertically DIFFERENTIATED OLIGOPOLY}

Copyright belongs to the author. Small sections of the text, not exceeding three paragraphs, can be used provided proper acknowledgement is given.

The Rimini Centre for Economic Analysis (RCEA) was established in March 2007. RCEA is a private, nonprofit organization dedicated to independent research in Applied and Theoretical Economics and related fields. RCEA organizes seminars and workshops, sponsors a general interest journal The Review of Economic Analysis, and organizes a biennial conference: The Rimini Conference in Economics and Finance (RCEF) . The RCEA has a Canadian branch: The Rimini Centre for Economic Analysis in Canada (RCEACanada). Scientific work contributed by the RCEA Scholars is published in the RCEA Working Papers and Professional Report series.

The views expressed in this paper are those of the authors. No responsibility for them should be attributed to the Rimini Centre for Economic Analysis. 


\title{
Product Line Pricing in a Vertically Differentiated Oligopoly*
}

\author{
George Deltas \\ Department of Economics \\ University of Illinois, U.-C. \\ Urbana, IL 61801 \\ United States \\ deltas@illinois.edu
}

\author{
Thanasis Stengos \\ Department of Economics \\ University of Guelph \\ Guelph, Ontario, N1G 2W1 \\ Canada \\ tstengos@uoguelph.ca
}

MAY 2010

\author{
ELEFTHERIOS ZaCHARIAS \\ Department of Economics \\ Athens School of Economics \\ Athens \\ Greece \\ ezachar@aueb.gr
}

\begin{abstract}
This paper empirically examines the joint pricing decision of products in a firm's product line. When products are distinguished by a vertical characteristic, those products with higher values of that characteristic will command higher prices. We investigate whether, holding the value of the characteristic constant, there is a price premium for products on the industry and/or the firm frontier, i.e., for the products with the highest value of the characteristic in the market or in a firm's product line. The existence of price premia for lower ranked products is also investigated. Finally, the paper investigates whether firms set prices to avoid cannibalizing the other products in their portfolio, whether competition with rival firms is stronger for products that are closer to the frontier compared to other products, and whether a product's price declines with the time it is offered by a firm. Using personal computer price data, we show that prices decline with the distance from the industry and firm frontiers. We find evidence that consumer tastes for brands is stronger for the consumers of frontier products (and thus competition between firms weaker in the top end of the market). Finally, there is evidence that a product's price is higher if a firm offers products with the immediately faster and immediately slower computer chip (holding the total number of a firm's offerings constant), possibly as an attempt way to reduce cannibalization.
\end{abstract}

J.E.L. Codes: L110, D430, L630.

Keywords: Pricing, Multiproduct firms, Personal Computers, Product Entry and Exit.

*We would like to thank Emek Basker, Matt Lewis, Chrysovalanta Milliou, the Editor, an anonymous referee, and seminar participants in the International Industrial Organization Conference for helpful comments and discussion. All errors are our own. 


\section{Introduction.}

Multi-product firms are the rule rather than the exception. Typically, each firm offers a number of products that are substitutes to each other and which also compete with similar products offered by rival firms. In such cases, firms do not price each product in isolation, but rather make joint pricing decisions for their entire product line. Moreover, the pricing of each product depends on the degree of competition that the product faces from product lines of rival firms. The relevant metric of competition is not merely the number of products that compete directly with a firm's particular product, but the entire product line produced by the rest of the industry. In the characteristics approach to product differentiation, products can be described by a list of observable characteristics; the relation among products of the same firm or those of a firm and rival firms can be made on the basis of a distance metric in the characteristics space. In many cases, these characteristics are of a "vertical" nature, i.e., they are such that consumer willingness to pay for a product is non-decreasing in these characteristics.

In this paper, we focus on aspects of product line pricing decisions in such vertically differentiated oligopolistic markets. Though the number of distinct characteristics is potentially large, we consider the case of a single salient vertical characteristic, or (equivalently) consumers who can arbitrage the values of all other characteristics by purchasing them as add-ons from third-party suppliers or from a separate menu. When there is a single salient vertical characteristic, a firm's products can be rank ordered on the basis of that characteristic from "highest" to "lowest," as can be the products of rival firms. One can then compare a firm's product line with that of the industry as a whole and, in particular, compare how close is each product of a firm to the industry frontier (i.e., to the best product available by all firms in the industry). Each product of every firm also has well defined "neighbors," i.e., products with the next higher or next lower value of the salient characteristic. Competition for a particular product, then, arises not only from similar products offered by rival firms, but also by its neighbors within the firm.

If there is a monopoly firm offering a menu of products, intuition would suggest that the order of a product in the product line would influence its price, all other things being equal. For example, 
introducing a new product with a value of the characteristic that is higher than all existing products, should (according to casual intuition) reduce the price of all the other products of the monopolist. Moreover, the introduction of a product may (according to similar intuition) increase the price of neighboring products as the firm would attempt to avoid cannibalizing the sales of one product by those of the other. Such thinking might be loosely based on Mussa and Rosen (1978) who show that monopoly mark-ups increase with product quality. However, Itoh (1983) has shown that this intuition is in fact false in the context of a pure vertical differentiation model. In particular, he has shown that, in such a model, the introduction of a product has no effect on the optimal price of lower quality products and has an effect of indeterminant sign on the optimal price of superior quality products (of the same firm). In the important special case of uniformly distributed willingness to pay for marginal increases in product quality, the introduction of a product has no effect on the price of all other products of the firm. Neither the introduction of neighboring products, not the order of a product in a firm's product line is relevant to its price. ${ }^{1}$

In vertically differentiated oligopolies, the effect of product introduction affects both the competition between firms as well as the relative position of products within the industry and firm portfolios. To our knowledge, there has been no theoretical work on multi-product vertically differentiated oligopolies that sheds light to product pricing relative to the location of the product in the firm and industry portfolios. ${ }^{2}$ One could attempt to identify the effect of a product location in the industry by considering single-product firms through a small modification of existing models in the literature. This can be done, for example, by considering a duopoly with zero production costs (to abstract from the effect that quality may have on marginal costs), and examining the effect on the price of the current high quality product from the withdrawal of the current low quality product and its replacement by an even higher quality product. To make the exercise meaningful, the qual-

\footnotetext{
${ }^{1}$ Itoh (1983) assumes that each consumer has a unitary demand for a product. However, one can show that these results continue to hold in an example in which each consumer has downsloping linear demand for the product, as long as they purchase a single variant of the product.

${ }^{2}$ In a very special setting, Siebert (2003) analyzed the optimal provision of goods in a market characterized by vertical product differentiation in a duopoly context. He shows that if one of the firms innovates by introducing a better product, it will always withdraw the existing product from the market in order to reduce price competition and to avoid cannibalizing the demand for its new product.
} 
ity of the new product must be such that a natural measure of competition between the products stays constant. In this environment, the price of the hitherto highest quality product goes down if consumer willingness to pay for product quality is uniformly distributed. This follows nearly immediately for fully covered markets from the results in Tirole (1988), or with some additional derivations for partially covered markets by modifying the analysis in Gabszewicz and Thisse (1979) and Shaked and Sutton (1982). In other words, in a stylized vertically differentiated single-product duopoly, the market leader commands a price premium relative to the market laggard. ${ }^{3}$ We should note, however, that this conceptual exercise is more similar to Mussa and Rosen (1978) than to Itoh (1983), where relative quality is conflated with the quality ranking.

There is also a small theoretical literature on mixed vertical-horizontal differentiation markets with multi-product firms. This literature focuses on product introduction and mark-ups, rather than on the price effects of relative position of products in a firm and industry portfolios. ${ }^{4}$ Katz (1984) shows that the introduction of a product of a particular quality by a firm can have negative cascade effects on the prices of the products of rival firms across the entire product range, and thus adversely affect the competitive environment. Therefore, firms have incentives to not produce full product lines in order to maximize the profits that they earn from firm-specific differentiation advantages in specific submarkets. In another related paper, Gilbert and Matutes (1993) show that in a symmetric equilibrium, all firms have the same mark-up for all their products (asymmetric sequential equilibria also exist). Barron, Taylor and Umbeck (2000) extend the modeling framework developed in the preceding two papers and focus on symmetric price equilibria. They consider the possibility that the substitutability between the products of rival firms is not the same across the quality spectrum, which naturally leads to a systematic relationship between mark-ups and product quality. In an empirical application, they show that the difference in price-cost margins between

\footnotetext{
${ }^{3}$ One can show that in fully covered markets the ratio of prices between the two products is independent of their relative qualities, i.e., it is purely a function of the product order (assuming that production costs are zero). In partially covered markets the price ratio is twice the ratio of the consumer willingness to pay for the two products, i.e., the market leader carries a price premium over the incremental value of the product to the consumers.

${ }^{4} \mathrm{~A}$ cross between these models and Mussa and Rosen (1978) is the recent contribution of Yang and Ye (2008). Individual firms can offer a continuum of qualities, but are fixed with respect to the horizontal characteristic in a Salop circle. The effects of competition are not unambiguous with regards to the quality/markup distortion for intermediate levels of competition, though a movement from monopoly to duopoly would reduce price-quality distortions.
} 
high and low quality gasoline prices depends positively on both the consumers' average valuation for incremental increases in quality and on the distance of each competitor's closest rival.

This paper estimates how the price of a firm's product is affected by the firm's product line and that of other firms. Our primary focus is on the relationship between a product's price and its location relative to the firm and industry frontiers, i.e., the relationship between a product's price and the number of products with higher attribute values that are offered by the same firm or by rival firms. We also investigate whether the availability by the same firm of products with similar attributes affects product pricing. Finally, we estimate how the number of competing products with the same attribute value impacts pricing across the product line. To obtain strong identification of any pricing effects relative to the firm and industry frontiers, it is important to examine pricing in an industry with rapid product turnover, both at the firm and the industry level, with frequent changes in the location of the industry and firm frontiers. The empirical analysis would also be greatly facilitated if the consumers strongly value one of the product characteristics, if other characteristics can easily be controlled for, and if there is relatively little unobserved heterogeneity within each brand's product offerings. As we discuss below, the personal computer industry in the 1990s satisfies all of these conditions, with the computer chip being the salient characteristic.

We find that computer prices are higher for the flagship products (highest speed computers) of a firm, even after accounting for the direct effect for higher performance on price. Prices decline as we move down the product line. A similar finding holds with regards to product location relative to the industry frontier. Conditional on location in a firm product line, prices are higher the fewer the number of products that are closer the industry frontier (again, after controlling for performance). More strikingly, for computers with any particular computer chip, prices are systematically higher the closer the computers are to the firm frontier (they are by definition equidistant to the industry frontier). Since these computers have identical processors, they are equivalent in terms of performance; however, they command higher prices if they are a firm's flagship product, somewhat lower prices if they are a firm's second fastest product, and even lower prices if they are the third fastest product. Firms try to limit cannibalization of their 
products by raising prices of a computer when they introduce a product that has the next slower or next faster processor; firms also lower a product's price the longer they offer it. Finally, topof-the-line prices of computers are sensitive to the number of firms that offer them; competition is somewhat less important for the second fastest chips in the industry, while it has no effect for even slower computers. Given the findings on average prices, this result suggests that firms earn rents for the high quality products, but prices for products of lower quality are nearly perfectly competitive: competition is stronger for low rank/quality products than for high quality products. Our conclusion that mark-ups are higher for products of higher relative quality than other offerings of the same firm (and that this effect is stronger the higher the rank of these products relative to industry offerings) can be interpreted as being supportive of the notions in Mussa and Rosen (1978) but not as consistent with the results in Itoh (1983) and Gilbert and Matutes (1993). Thus, our results can have implications for the applicability of vertical differentiation models and also provide insights for the degree of substitutability between various components of rival product lines.

Two other empirical studies have observed results consistent with our findings that competition is stronger for lower quality products, and that markups are higher for products high in the quality ladder. These studies have weaker identifying power to isolate the effects of relative product positioning and the authors attach somewhat different interpretations to their findings. In an analysis of the auto industry, Verboven (1999) finds that the absolute mark-up on base models is lower than the absolute mark-up on premium models. He attributes this finding to limited consumer information, in which consumers are well informed about the base models but not about the premium models. An alternative interpretation is also possible: that consumers who purchase the premium models have a higher willingness to pay for the brand of their choice, i.e., that they are characterized by stronger brand preferences or brand loyalty. ${ }^{5}$ Manez Castillejo (1999) investigates the intensity of price competition across the quality gradient. He finds that price

\footnotetext{
${ }^{5}$ This possibility is not considered in Verboven (1999). Indeed, using a simple model, he shows that in symmetric competition between product lines, mark-ups are the same for high and low quality quality products if consumers are perfectly informed about product attributes. But built into the model is the assumption that consumers with high willingness to pay for premium products have equally intense brand preferences as those with low willingness to pay for premium products. In a less related paper, Verboven (2002) finds that European auto manufacturers price discriminate on the basis of usage intensity across the gasoline and diesel variants of their car models.
} 
competition is weaker for higher quality products (branded products or high quality store brands) and stronger for lower quality products (low quality store brands). The analysis can be construed as a confirmation of the view that the intensity of price competition is greater for those quality variants with less possibilities of horizontal product differentiation (an interpretation adopted by the author). Alternatively, we believe that it can an indication that high value consumers, who are more likely to purchase high quality goods, are characterized by stronger brand preferences.

Our dataset allows for a sharper identification of the effects of relative quality because each product is observed at many different relative positions, and because there is substantial product turnover. Moreover, price information is nearly perfect. Thus, unlike in the case of automobiles analyzed in Verboven (1999), it is not tenable to assume that the higher mark-ups in high quality products are driven by imperfect price information of consumers. Similarly, unlike the case of Manez Castillejo (1999), it not tenable to assume that differences in mark-ups are driven by differences in product differentiation, since in our results we are able to compare prices of the same product in different firm and industry relative locations, and since we also control for all meaningful product attributes. Thus, we attribute the systematic differences in pricing to differences in consumer preferences across different parts of the market spectrum. In this sense, our results are closer in spirit to the empirical findings on gasoline pricing in Barron, Taylor and Umbeck (2000), with an important distinction being that we use intertemporal variation instead of spatial variation in product offerings as the source of identification. ${ }^{6}$

\section{Data and Variables.}

\subsection{Identification-Relevant Features of the Dataset}

The data consist of prices of personal computers sold in the BuyDirect market segment during the mid 1990s. During this period, personal computers were almost fully described by their price

\footnotetext{
${ }^{6}$ There is an alternative behavioral explanation that follows the reasoning in Ariely, Loewenstein and Prelec (2003). If consumers do not have a direct measure of value for computers of different capabilities, then their willingness to pay could be influenced by anchors. The price of the flagship product can be such an anchor, and if set high, it could lead consumers to perceive lower capability computers as good bargains. Such behavior would create incentives for firms to have high markups for their top products.
} 
and a list of characteristics. There was limited (if any) unobserved heterogeneity that is not captured by the brand identity, and in particular limited attempt to differentiate on the basis of design or other non-quantifiable features. Moreover, except for the processor, essentially als other computer features were offered as an add-on with a menu of different options available by each manufacturer (or as an upgrade by a third-party firm). ${ }^{7}$ A computer's processor was the single most important characteristic (in fact, the one around which price quotes with different options were organized). It was also the one characteristic that it was impossible to "upgrade" without replacing the computer itself. Thus, once the prices of the other features of each listing are controlled for, personal computers in that time period can be described as a vertically differentiated product with the processor capability being the salient vertical characteristic. This does not imply that there is zero differentiation beyond the processor type. Brand can be thought of as a characteristic that imparts an element of horizontal differentiation. We thus treat this as a product market in which (after controlling for all the other computer features), products are distinguished vertically by the processor capability and horizontally by the brand that offers them.

Another important feature of this market and time period is that there is wide intertemporal variation in the number of products offered by the industry, with many processors being introduced by Intel and others being retired. Firms did not introduce or discontinue offerings with each particular processor at the same date, nor did they offer equally broad product lines, or even make the same choices about which processors to offer conditional on the breadth of the product line. Thus, there is substantial variation in the position of each processor in the industry product order; moreover, conditional on the industry product order, there is substantial variation in the position of each processor in the firm product order. This rich variation allows for an easy identification of the product line order effects and (as it turns out) estimate them rather precisely even when controlling for all other effects semiparametrically. The identification is greatly facilitated by the fact that the

\footnotetext{
${ }^{7}$ The important point is whether the pricing structure takes this upgrade possibility into consideration, or (more generally) whether the pricing structure is of the form of "price of base product + price of add-ons." This indeed is the case. Moreover, the price of incremental features is independent of base model that they are added to. Thus, the price differences between any two models are largely driven by price differences in the processor they embody.
} 
feasible set of processor capabilities available to the computer manufacturers is exogenous to them, as they all offered chips made by Intel. Had this not been the case, and had instead each firm made its own set of processors, it would not have been possible to compare processors that had the same capability and order in the industry line up but differed in their position in the firm line-up.

It is important to emphasize that the variation in the data allows us to identify the effect of product order without relying on functional form assumptions with respect to the impact of processor speed on price. In other words, the premium on being on the firm and industry frontier does not (in principle) pick up non-linearities of the hedonic pricing function with respect to processor speed. The effects of a product's location in a firm's product line are identified from the comparison with identical products of other firms that are in different location in the product lines of those other firms. For example, the price effects of being the fastest product of a firm versus being the second fastest product of a firm are identified by comparing prices of identical products that are the fastest offering in some firms and the second fastest offering in other firms, adjusting flexibly for all other product features including brand fixed effects and the date of the product listings. ${ }^{8}$ The effect of a product's location relative to the industry frontier is identified through the change in the set of product offerings in the industry over time. ${ }^{9}$ For any hedonic function that relates processor speed to computer price, the effect of location with respect to the industry frontier is obtained by comparing the price of a processor before and after the introduction of other processors, allowing for linear or multiplicative shifts in the hedonic relationship across time periods (and controlling flexibly for all other product characteristics). In other words, the price premium for computers that carry the fastest chip in the industry versus computers that carry the second fastest chip in the industry is estimated from the change in the price of computers that were the fastest on offer in time $t$ after an even faster computer is introduced in time $t+1$. This comparison is made after controlling for the fact that all prices change from $t$ to $t+1$ by a given percentage, and adjusting

\footnotetext{
${ }^{8}$ In principle, this effect can be identified solely by comparisons of products that are sold in the same point of time, but across time period information is also utilized in obtaining the estimates (and is in practice necessary).

${ }^{9}$ There was a total of 13 distinct chips offered during this time period. None of the four processors available at the start of our sample $(25,33,50$ and $66 \mathrm{MHz} 486$ chips) were available at the end of the sample. Moreover, four chips introduced during the sample period were discontinued by the end of our sample. There is a large variation in the location of a chip relative to the industry frontier.
} 
for all other characteristics. ${ }^{10}$

Finally, it is important to emphasize that our focus in not the price/quality relationship (or price/mark-up relationship). Though this is the focus of some of the theoretical literature (e.g., Mussa and Rosen 1978), there is a fundamental obstacle in relating the theory with hedonic regression results: what is labeled "quality" in the theory cannot simply be replaced by "clock speed" in the empirics, even if that were the only computer characteristic. In the theory, quality is often defined as a suitably normalized vertical characteristic so that a consumer has a constant willingness to pay for each incremental unit of it; but there is no reason to believe that consumers have a constant willingness to pay for incremental changes in clock speed. Clock speed relates to quality through some monotonic function with in principle unknown properties. Thus, the theoretical relationship between the price and speed is also dependent on this same function's properties. An additional challenge when focusing on the relationship between mark-up and speed is that in hedonic regressions, costs are not observed. Our analysis, which compares prices of computers with the same physical characteristics under different product menu configurations using a difference-in-difference approach, side-steps all these issues.

\subsection{Sources and Variables}

Our source of data is the PC Magazine. This is one of the leading magazines for personal computers and thus, it is the main source of price information for the buy direct segment of the market. We collected data on the first issue of every month from January of 1993 to December of 1995. These are the last three years for the 486 processors. Pentium processors are introduced around the middle of this period. Each observation consists of the advertised price and features of a personal computer. We limited our attention to price quotes of the following 10 firms: Acer, Austin, Compaq, Comtrade, Dell (excluding the Optiplex models since they are targeting the business market) Gateway 2000, IBM, Micron, Midwest Micro, and Zeos, since these are some of the most frequently advertised firms. The price quotes of these firms contain the overwhelming majority of price quotes by manufacturers

\footnotetext{
${ }^{10} \mathrm{It}$ is worth mentioning that not all new chips are the fastest available at the point of their introduction. Occasionally, a new chip is introduced to "backfill" the product space (the $75 \mathrm{MHz}$ Pentium being a prominent example).
} 
in PC Magazine. For this segment of the market, list prices are equal to transactions prices for purchases by individual consumers.

In every advertisement, each firm lists the price and all the features for a number of models. In a number of cases, in the same advertisement, the firm also lists the incremental price of upgrading some features on these "base" models. Every possible combination that involved an upgrade of a major component has been used to create a separate observation. Moreover, we also included minor upgrades that incorporated a specific minor feature when this was also associated with an increase in the price, even when it where not controlled in our regressions. Incorporating these upgrades contributes essentially to the error term, as the value of the right hand side variables is the same for these observations. Our raw data-set consists of 10,450 observations. ${ }^{11}$ During the period we examine, the firms we consider advertise 6 different 486 processor types $(25 \mathrm{MHz}, 33 \mathrm{MHz}, 50 \mathrm{MHz}$, $66 \mathrm{MHz}, 75 \mathrm{MHz}$ and $100 \mathrm{MHz})$ and 7 different Pentium processor types $(60 \mathrm{MHz}, 66 \mathrm{MHz}, 75 \mathrm{MHz}$, $90 \mathrm{MHz}, 100 \mathrm{MHz}, 120 \mathrm{MHz}$ and $133 \mathrm{MHz}$ ). It is worth noting that not all firms offer each processor type every month. The time period that each processor is advertised as well as the number of price listings for different processor types varies across firms. In a small number of cases, a firm was not observed to advertise a particular processor in a given month, but was observed to advertise it in the preceding and following months. We make the reasonable assumption that the processor was continued to be available during that intervening month (and consumers could presumably purchase it by using the information in the ad of the preceding issue of PC Magazine). This assumption does not generate additional observations, but affects (in a limited way) the values of the variables that provide information about a firm's product portfolio.

An observation consists of the price of a computer $(P)$, its associated features, time-related controls, and product line related information. The product characteristics are: the clock speed of the processor in $\mathrm{MHz}(S P D)$, an indicator variable for whether the processor is a Pentium chip $(P E N T M)$, the size of the hard drive in $\mathrm{MB}(H A R D)$, the size of the RAM in $\mathrm{MB}(R A M)$,

\footnotetext{
${ }^{11}$ For example, some models were offered either in desktop or tower form, sometimes at the same price and sometimes at a different price. For a more detailed description of the data set see also Deltas and Zacharias (2004, 2006).
} 
indicator variables for the size of the screen (15 inch and 17 inch, SCRN15 and SCRN17, 14 inch being the omitted category), an indicator for the presence of a $\mathrm{CD}-\mathrm{ROM}(C D)$, an indicator the presence of a multi-media kit that includes speakers and a sound card $\left(M U L T I_{i}\right)$, a dummy indicating the identity of the manufacturer $\left(A C E R_{i}, A U S T I N_{i}, C O M P A Q_{i}, C O M T R A D E_{i}\right.$, $D E L L_{i}, G A T E W A Y_{i}, I B M_{i}, M_{C R O N}, M I D M I C R O_{i}$, and $\left.Z E O S_{i}\right)$ with $D E L L_{i}$ being the omitted dummy), and a dummy indicating the month $t$ of the price listing. In the regression analysis, we use a number of interactions between the most important of these variables, as well as (in some specifications) interactions between the time trend and its powers with the product characteristics. When the regression is estimated in log-linear rather than in linear form, $P, H A R D$, $R A M, S P D$, are replaced by their natural log.

The product line related information for each observation consists of (i) a series of indicator variables which indicate the order of the product in the industry offerings and the firm line-up, with variable $F R M$ foINDio indicating that product is the $i o^{\text {th }}$ fastest in the market in the month that corresponds to that observation and the $f o^{\text {th }}$ fastest among the firm's product offerings in that same month, with market positions fourth and below lumped together and firm positions fourth and below forming the omitted category, (ii) an indicator $(A B O V E)$ that takes the value of 1 if a firm offers the next fastest chip in the same month, (iii) an indicator $(B E L O W)$ that takes the value of 1 if the firm offers the next slower chip in the same month, (iv) the number of different processors offered by a firm in that month $(F R M D L S)$, (iv) the number of months that a firm has been offering products containing a particular processor (TFIRM), (v) the number of rival firms offering this same chip (NSMCHIP) in that month and the interactions of this variable with indicator variables for the fastest and second fastest chips in the market (NSMCHIP1 and NSMCHIP2). Because the relevant comparisons of computer prices for products in order 1 through 3 in a firm's line-up is only meaningful relative to products ranked fourth or below, we drop all observations of a firm in a month if in that month that firm offered less than four different processor speeds. This reduces are sample somewhat to 9,339 observations. However, the dropped observations are still utilized in the construction of the industry offerings and the degree of competition for each chip, 
as we outlined above. In constructing the order of a chip with a firm's and an industry's offerings, we use the processor type and processor speed. All Pentium computers are considered of higher order than all 486 computers, and within each processor type, the higher the speed, the higher the order. Note that some Pentium chips are of lower clock speed that some 486 chips. However, in benchmark tests, Pentium chips were performing at nearly twice the level of a 486 chip with the same clock speed. If the clock speed of the Pentium chips was adjusted by a factor of 1.9 that is consistent with benchmark tests, the Pentium with the slowest nominal clock speed would indeed have a higher effective clock speed than the fastest 486 chip. ${ }^{12}$ We emphasize that we do not scale the clock speeds in any of the regressions, but rather estimate different coefficients for the Pentium and 486 computers for a number of variables, including computer clock speed.

Table 1 provides some key summary statistics on the distribution of observations across the key product line related variables, and the associated average prices. Observe that the fastest chip offered by most firms in a particular month is also the fastest available in the market. However, a firm's fastest chip on offer is not the fastest chip in the market in one quarter of the cases. In fact, in 37 observations, a firm's fastest chip is not among the three fastest on offer in the market. Similarly, there exists substantial variation in the position of the second and third fastest chips of firms relative to the set of products offered in the industry. The average prices of offerings for each of the positions exhibit some systematic pattern. Holding the rank in a firm's product line-up fixed, computers tend to be sold at lower prices the lower their rank in the market. Similarly, holding the rank in the market fixed, prices tend to be lower the lower the computer's rank in a firm's portfolio. However, this relationship is not monotonic and is confounded with many other very important factors; it is instructive, though, that some reasonable relationship between product location and prices emerges even without formal regression analysis. In about two thirds of the observations, a firm offers a product that contains the immediately faster and immediately slower chip. The price

\footnotetext{
${ }^{12}$ We obtained some econometric evidence consistent with this clock speed scaling. In standard hedonic regressions that utilize only computer characteristics, multiplying the clock speed of Pentium computers by 1.9 results in a statistically insignificant dummy for Pentium computers. In other words, in order to remove the observed price premium of Pentium computers in such hedonic regressions, the clock speed of the Pentium computers must be scaled up to the level that the slowest Pentium is computationally faster than the fastest 486.
} 
of those chips for which the firm offers the immediately faster chip is lower than those for which the firm offers the immediately slower chip, but only because the former tend to be (by definition) on average of lower rank in the product line than the latter.

Table 1 also shows that on average there are almost six (out of nine) rival firms that offer computers with any given chip. The number of competing firms offering the fastest chip in the industry $(0.7 / 0.1232 \approx 5.7)$ and the second fastest chip in the industry $(1.05 /(0.0362+0.1404) \approx$ 5.9 ) is approximately the same. This, of course, does not imply the competition is equally intense for the fastest chips in the industry as it is for the average chip in the industry. If the customers who purchase the frontier products have stronger brand preferences, competition would be less intense for that market segment. Finally, each firm offers (in the typical month) a little over six distinct chips in its product line.

\section{Econometric Framework}

Our analysis adopts an augmented flexible hedonic regression approach. We recognize that the price of a personal computer will depend largely on its physical characteristics, the time period it is offered for sale, and its brand. These are standard ingredients in a hedonic regression model. We augment the model by including product line information, which is the focus of this paper. We treat all the "standard" components of a hedonic regression as nuisance parameters: we are not interested in their values, but want to ensure that they do not contaminate our parameters of interest. Because the marginal effects of computer characteristics may vary over time, we allow (in most specifications) all the parameters that correspond to physical characteristics to be a polynomial function of time. This is achieved by multiplying all the computer characteristics with powers of the monthly trend. Though this model is still parametric, it exhibits sufficient flexibility with regards to the price effects attributed to the computer characteristics that we expect it to account for their contribution to the price. We estimate both linear and log-linear models, not only in order to verify that the conclusions are robust to specification, but also because they naturally obtain estimates in terms of average effect in dollars and average effect in percentage terms, respectively. 
We provide below the general specification for the log-linear model; in the linear counterpart, none of the variables are in logs. Our benchmark parametric specification is given by the regression

$$
\begin{aligned}
\ln \left(P_{i, t}\right) & =\sum_{f o=1}^{3} \sum_{i o=f o}^{4} \alpha_{f o, i o} F R M f o I N D i o_{i, t}+\beta_{A} \text { ABOVE } E_{i, t}+\beta_{B} \text { BELOW }_{i, t}+\beta_{F} F R M D L S_{i, t} \\
& +\beta_{N} N S M C H I P_{i, t}+\beta_{N 1} N S M C H I P 1_{i, t}+\beta_{N 2} N S M C H I P 2_{i, t}+\beta_{T F} \text { TFIRM } M_{i, t} \\
& +\sum_{l=0}^{L} t_{i}^{l}\left(\gamma_{H, l} \ln \left(H A R D_{i}\right)+\gamma_{R, l} \ln \left(R A M_{i}\right)+\gamma_{S, l} \ln \left(S P D_{i}\right)+\gamma_{S P, l} P E N T M_{i} \ln \left(S P D_{i}\right)\right) \\
& +\sum_{l=0}^{L} t_{i}^{l}\left(\gamma_{C, l} C D_{i}+\gamma_{M, l} M U L T I_{i}+\gamma_{D 15, l} S C R N 15_{i}+\gamma_{D 17, l} S C R N 17_{i}\right) \\
& +\sum_{b}^{9} \sum_{l=0}^{L} t_{i}^{l}\left(\gamma_{b, l} B_{b, i}+\gamma_{b P, l} B_{b, i} P E N T M_{i}\right)+\sum_{t=2}^{36} \tau_{t} t_{i}+\sum_{t=10}^{35} \tau_{P, t} t_{i} \operatorname{PENTM}_{i}+\varepsilon_{i, t}
\end{aligned}
$$

where $L$ is the order of the time polynomial, $B_{b, i}$ is a set of nine brand dummies (one of ten firms is excluded), $\tau_{t}$ are the coefficients on the set of time dummies, $\tau_{P, t}$ are the coefficients on the interactions of the time dummies with the Pentium dummy for the periods both Pentium and 486 computers are offered for sale and $\varepsilon_{i, t}$ be a disturbance term that is orthogonal to all regressors. ${ }^{13}$ Heteroskedasticity consistent robust standard errors that allow for clustering at the firm-chip-month level are computed and reported. ${ }^{14}$ In our analysis, these turn out to be uniformly more conservative (larger) that either the OLS standard errors or the heteroskedasticity consistent standard errors with no clustering. We have estimated the model for various values of $L$ (including $L=0$ ) and for various sub-sets of the parameters included above. Our "base" model uses a time polynomial interaction of order $L=2$, resulting in 153 estimated parameters (including the constant). ${ }^{15}$

We complement the above polynomial parametric regressions with a semi-parametric analysis, which consists of the following procedure. We estimate for each observation $j=\{i, t\}$ in the sample the regression model given in equation 1 using $L=2$ and weighting every observation $j^{\prime}$ by $w_{j^{\prime}}=\sqrt{\phi\left(\frac{\left|t_{j^{\prime}}-t_{j}\right|}{h}\right)}$ where $\phi(\cdot)$ is density of the standard normal and $h$ is the bandwidth. In

\footnotetext{
${ }^{13}$ A single dummy from the first set is dropped to avoid collinearity.

${ }^{14}$ Observations that correspond to the product offerings of a particular firm that contain a particular chip during a particular month are assumed to have correlated disturbance terms. Following the approach of White (1980) no assumptions are made about the nature of heteroskedasticity. See Wooldridge (2002) for further details.

${ }^{15}$ The set of parameters in the model is actually equal to 155 , but two interactions involving the Compaq dummy are being dropped. This also indicates that further functional form flexibility comes at the cost of losing some useful information.
} 
the regression minimization problem, this is equivalent to weighting the residual associated with observation $j^{\prime}$ by $w_{j^{\prime}}^{2}{ }^{16}$ For the "base" semi-parametric analysis, we choose a bandwidth of $h=10$. For this bandwidth, the residuals of observations that are one year away from observation $j$ have a weight that is slightly less than half that of the residuals of observations that are contemporaneous to observation $j$. The residuals of observations that are more than two years away from that of observation $j$ have weights that are five percent or less of the weight of residuals of observations contemporaneous to $j$. Loosely speaking, each of the locally weighted regressions uses (in terms of "weighted" observations), about a quarter of the sample information. For robustness, we have also estimated some regressions with the much smaller bandwidth of $h=5$ and higher polynomial time trend interactions (up to $L=4) \cdot{ }^{17}$

These locally-weighted regressions result in a set of observation-specific parameter estimates. Denote the observation specific estimates of parameters $\alpha_{f o, i o}$ and $\beta_{m}$ by $\alpha_{f o, i o, j}$ and $\beta_{m, j}$, respectively. Following Zhang, Lee, and Song (2002), our estimates of $\alpha_{f o, i o}$ and $\beta_{m}$ are given by $\alpha_{f o, i o}=\frac{1}{N} \sum_{j=1}^{N} \alpha_{f o, i o, j}$ and $\beta_{m}=\frac{1}{N} \sum_{j=1}^{N} \beta_{m, j}$, respectively. Standard errors are obtained by a block bootstrap with resampling of groups of observations that consist of all product offerings of a firm in a given month that contain a particular processor (e.g., all $100 \mathrm{MHz} 486$ computers offered by IBM in January of 2004 would consist one such block). This block bootstrap accounts for the fact that all these observations share a common "shock" to their prices, even after accounting for the rich variable set in our regression. The normal approximation is used to determine significance on the basis of these standard errors.

The above specifications utilize both time-series and point-in-time variation in the data. One way to utilize only point-in-time variation to identify the main effects is to interact the main effects with time dummies and use the average value of the point estimates for each of the 36 months as the summary measure of each main effect. Since 9 variables are of greatest interest (the

\footnotetext{
${ }^{16}$ Internal to the estimation routine, we normalize the weights so that the average weight is equal to 1.

${ }^{17}$ This procedure is a modification of Zhang, Lee, and Song (2002) and Stengos and Zacharias (2006), who divide the variables into a linear and non-linear group. In our implementation, the parameters of interest are those of the linear group. We modify their approach by using a fixed bandwidth and polynomial order, and estimating the regression using a number of different bandwidth and order values. This reduces computational burden and permits us to compute standard errors via a block bootstrap, which mimics cluster-based standard errors.
} 
ones that describe a product's position relative to the industry a firm frontier), this would lead to the addition of approximately 330 new variables. This is not feasible because many of these effects are identified from a very small number of observations, e.g., there are only 37 observations for which $F R M 1 I N D 4_{i, t}=1$. There are many months for which a number of data "cells" are completely empty and point estimates for the interactions are unobtainable. Other cells that are not completely empty have too few observations to provide credible estimates. One approach that moves half-way towards relying on point-in-time variation is to interact the variables of interest with quarterly dummies (instead of monthly dummies), and use the average of these quarterly dummy interactions to obtain the effect of interest. We perform this interaction with the most populated cells, which correspond to the models that have the same rank in both the industry and firm frontiers. This results in $3^{*} 12=36$ interactions (an addition of 33 variables to equation 1 ).

We note that all estimation approaches take the product offerings as exogenously given. As in all hedonic models, a bias could result if unobserved product characteristics are correlated with observed product characteristics, and (more importantly for this paper) with product location relative to the frontier. We do not believe it is an important concern for two reasons. First, unobserved characteristics at the product level are extremely unlikely to change at a monthly frequency. Such characteristics include the computer case, possibly the graphics memory card, the type of keyboard, and other minor features. Some of these features do not differ at all across computers of the same brand and they are captured by the time-varying brand dummy. Some that might vary across models of the same firm (e.g, the type of graphics memory card), are fixed for a particular model. Second, if these unobserved characteristics were to change for a particular model, they would improve, not worsen. If they were indeed to improve over time, then any bias this would induce would go against finding a price premium for frontier models: since models tend to move down from the firm and industry frontiers after they get introduced, an improvement in their unobserved characteristics as time passes would soften the decline in their relative prices. 


\section{Results}

The results of the baseline models are reported in Table 2. We first report (in column 1) the results of the simplest regression one could estimate using our variable set. This corresponds to a value of $L=0$ in the equation 1 with kernel weights equal to 1 for every observation. Focusing on the coefficient set FRM1INDio (the fastest computers offered by a firm), we see that these command a substantial price premium (after controlling for the clock speed and processor type) over the fourth or slower computers offered by the same firm. This price premium depends on how far these computers are from the industry frontier in terms of computing power, i.e., how many faster chips are available in the market. When the fastest product of a firm is also among the fastest available in the market, the price premium relative to the low-speed offerings of that firm is the highest (around 17\%); when it is the second faster in the market, the price premium is about 14\%; when it is the third fastest in the market, the price premium drops to around $10 \%$, while if it is not among the three fastest chips in the market, the price premium is only around $6 \%$. With regards to the second fastest product of a firm, when it is the 2nd fastest in the industry (by definition, it can never be the fastest in the industry), its price premium is $11 \%$, when it is the 3rd fastest in the industry, its price premium is $6.7 \%$, while when it is not among the three fastest in the industry, the price premium is $5.6 \%$. Prices decline monotonically with location among the industry offerings. The same pattern continues to hold when examining the third fastest computers offered by firms.

One might speculate that the relationship between price and order of a chip in the industry offerings might be driven by the pricing of Intel, the upstream input supplier. It could very well be that Intel chooses to reduce the price of a chip when it introduces a faster chip in the market (over and above any secular changes in the price of its offerings). In other words, it could be that the price gap between a fast Intel chip and a very slow Intel chips drops when an even faster Intel chip is introduced in the market. Such a price pattern would not nullify the premise that the order of a product in a vertically differentiated product line is informative about its price; it would just mean that the relevant product line is that of Intel and not of the computer manufacturers.

To identify effects that arise from computer manufacturer pricing decisions, one needs to look at 
the price of the chips that are in the same position relative to the industry offerings but in different positions relative to a firm's offerings. There is indeed a monotonically declining relationship between the processor order within a firm's offerings and price, holding the processor's order in the market constant. The computers that are the second fastest in the industry and fastest among a firm's offerings are more expensive than those which are the second fastest both in the industry and in the firm's offerings. The price difference is equal to a fraction of approximately $0.1365-0.1097=$ 0.0269 of the computer price. The effect is even more pronounced when we consider computers that contain the third fastest chip in the industry. Among these computers, those that are the fastest offering of a firm are more expensive than identical computers that are the second fastest offering of a firm by a fraction of $0.1008-0.0664=0.0344$ of the price. In turn, the second faster computers in a firm's portfolio are pricier than identical computers that are the third fastest in firm's portfolio by a fraction of $0.0664-0.0497=0.0168$ of the price.

With regards to the other product portfolio and competition variables, one statistically significant finding is that the price of a computer is higher if the firm also offers computers with the next faster or next slower computer chips: the coefficients of $A B O V E$ and $B E L O W$ are around 2 percent of the computer price. Another statistically significant finding is that prices tend to decrease with the number of firms that offer the same computer chip, but only for computers that are the fastest available in the market. Finally, the longer a particular chip is offered by a firm, the lower its price, relative to that of the same chip offered by other firms in the same point of time. The number of computers advertised by a firm appears to have no effect on prices.

A concern with the specification described above is that the contribution of product characteristics to the price of personal computers is likely to change over time. For example, the marginal cost of hard drive capacity, either in dollars or as a fraction of a computer's price, is unlikely to be constant over our sample period. If these changes are systematically related to any of the product portfolio variables (a real possibility if there are trends in the portfolio variables, e.g., if it becomes more likely over time that the representative firm offers or does not offer the fastest available chip, or if brands that command a higher price premium are systematically more or less likely to offer the 
fastest available chip, etc.), then biases could ensue. Though we would be unable to sign the bias on a priori grounds, this raises the possibility that any findings are partially contaminated by it. To reduce this possibility, we estimate and report (in columns 2) a model in which all product characteristics (including the brand dummies) are interacted with the time trend and the trend-squared. This corresponds to a value of $L=2$ in the equation 1, while kernel weights are still fixed to 1 for every observation. The polynomial trend interactions with the product characteristics are jointly statistically significant, despite the large increase in parameters. Moreover, standard errors of the parameters of interest tend to decline. These suggest that these polynomial time trend interactions do indeed capture systematic patterns in the data and improve our inference.

Comparing the results of this second set of regressions with the first set, we observe a few differences. The price premia of the 2 nd and 3rd fastest chips in the industry (regardless of location in a firm line-up) are somewhat smaller, with the reduction in the point estimates being particularly large for 3rd fastest chips in the industry. This strengthens our findings that prices decline with industry order from the fastest through the 3rd fastest chips, but leads to a couple of (statistically insignificant) "reversals" when comparing computers that are the 3rd fastest in the industry with those that are slower yet. One interpretation of this is that order effects are exhausted when we reach computers with the 3 rd fastest chip available in the industry. The competition effects are now noticeably bigger and they are (negative) statistically significant not only for computers that are the fastest in the industry, but also for computers that are the second fastest. Top-of-the line computers are priced at least 1 percent less for each additional competitor who offers a computer of the same clock speed. The corresponding effects for computers that are the second fastest in the industry is about half this size. Competition seems to have no effect for slower computers. Finally, under this (and the rest of the log-linear specifications), the length of time that a firm offers a particular chip appears to have a stronger negative effect on its price.

In the third model, we re-estimate semi-parametrically the above specification using locallyweighted regressions to obtain observation-specific parameter estimates. ${ }^{18}$ We report the average

\footnotetext{
${ }^{18}$ We employ a bandwidth of $h=10$ which results in following weight patterns. Data that is within six months of
} 
values of the parameters of interest in column 3. One of the first observations when comparing the locally-weighted (semi-parametric) results with those of the unweighted regressions is that the standard errors are now higher, sometimes dramatically so (e.g., consider the standard error of the coefficient of FRM1IND4). This suggests that the increased functional form flexibility comes at a cost of precision. Second, the point estimates for the product order parameters are very similar: generally smaller, but by very small margins. Lastly, the point estimates of the other product portfolio and competition parameters are essentially the same.

In the last of our base models, we provide estimates that are more heavily based on within time than across time variation. This analysis generates coefficients for the $F R M x I N D x$, where $x=1,2,3$ that vary by quarter. Since the individual estimates are not of interest, we report here the point estimate of the average over the 12 quarters, and the associated standard errors. These standard errors are higher their counterparts in the previous models, but the estimates are not systematically different (and neither are those for the other parameters).

We have re-estimated these models in linear, rather than log-linear form, obtaining estimates of the various effects in dollars rather than in percentages. The pattern of results is the same as the log-linear regressions, and provides us with additional assurance that findings are not driven by functional form. We report the linear counterpart of the 2 nd model as a representative sample.

To verify the robustness of our results, we perturb the regressions reported in Table 2 in a number of directions, but discuss rather than report the results in the interest of saving space. The first variation involves simplifying the most parsimonious model of Table 2 by dropping all product location and competition variables except the set FRM foINDio. There are number of concerns with dropping these variables arising from the fact that they are likely correlated both with the price and with the position of a product on the firm/industry frontiers and capture economically distinct price-relevant effects. Nonetheless, this simpler model is useful in investigating the extent to which our findings rely on the inclusion of these variables. The point estimates of the positional

a particular observation receive (on average) a weight equal to $95 \%$. Those within a year but more than six months receive a weight of $69 \%$, those in the next six months an average weight of $35 \%$, and the for successive six month intervals the average weights drop to $13 \%, 3 \%$ and nearly zero percent. 
premia for this model are somewhat smaller than those reported in Table 2, but they continue to be strongly statistically significant and exhibit the same declining pattern as we move down the industry order (holding firm order constant) or firm order (holding industry order constant).

Our second direction in terms of increased parsimony focuses only on computers that are the fastest and 2nd fastest among a firm's offerings. In these regressions, we include a larger set of observations as we only drop the observations that correspond to firms offering fewer than 3 (rather than fewer than 4) different processors in a given month. For this set of models, the estimates of the remaining product location variables are somewhat smaller than those in Table 2, a finding that is expected given that the 3rd fastest computers are now "pooled" with slower computers. However, the pattern of estimates is unchanged as are the estimates of the remaining product location and competition variables.

Our final robustness check is to estimate an even more flexible model than those reported in Table 2. We have increased the polynomial order of trend interacted with the physical product characteristics and brand dummies to a cubic and decreased the bandwidth to 5 . The results are similar to those with the higher bandwidth and lower polynomial order, though standard errors become higher. This increase in standard errors suggests that the increased flexibility reduces the amount of information used to estimate the parameters of interest without capturing additional important features of the data. The pattern of coefficients remains the same if we push the polynomial order to 4 (leading to yet higher standard errors), or increase the bandwidth at 10 . These additional regressions provide re-assurance that our findings are stable across specifications, even when we push towards specifications that are less appropriate than those reported in Table 2 .

\section{Discussion and Concluding Remarks}

One can summarize the regression results by averaging out the coefficients and reporting the implications for product prices. We do this in Table 3 for the four log-linear specifications. The results are presented in a matrix form, allowing for an easy comparison of prices of computers of a particular order in a firm's portfolio as a function of their order among the industry offerings, and the 
prices of computers of a particular order among the industry offerings as a function of their position in a firm's portfolio. The figures in panel A show that: (i) the price premium of a firm's product declines as its position drops relative to the industry frontier, and does so at a progressively smaller rate the further behind the frontier the product is located (with an occasional minor "reversal"). (ii) the price of a computer with a particular processor declines monotonically with its ranking in a firm's product portfolio, with the decline being generally bigger when the computer drops from being a firm's flagship product to being a firm's second fastest product.

The second result is the cleanest in terms of magnitude and pattern as we move to computers of lower order. Combined with the results on the other product portfolio and competition variables, these findings suggest that computer firms have been able to exercise some (second degree) price discrimination against consumers of high performance computers who attach a value from purchasing from those firms. This price discrimination is manifested in higher prices for a firm's flagship (or relatively high performance) computers relative to the firm's other product offerings, even after controlling for the product's absolute and relative (to the other industry products) performance. However, the premium for a firm's flagship product is smaller the further away this product is from the industry frontier. Moreover, firms are cognizant of the fact that introducing a particular product can cannibalize sales of other similar products that they offer. As a result, prices of a product are higher if it is adjacent in terms of performance to other products the firm offers, whether these products are of higher or of lower performance (though the result is stronger and consistently statistically significant if the adjacent product is of lower performance). This finding is hard to reconcile with results from a standard vertical differentiated monopoly model, and suggest a richer framework. ${ }^{19}$ Finally, the decline in a computer's price with the time it is offered by a firm, relative to the price of other identical computers, provides evidence of inter-temporal price discrimination at the brand level (and thus of brand differentiation). As in many models of dynamic monopoly, firms charge a high price initially and sell to the high value consumers (who have an attachment

\footnotetext{
${ }^{19}$ We recognize that the hard prediction of Itoh (1983) is that the coefficient of $A B O V E$ should be zero. According to the theory, the coefficient of $B E L O W$ can be of either sign (it is zero for uniformly distributed willingness to pay).
} 
to their brand), and lower prices of the product over time.

The number of firms offering a particular product also impacts pricing, but this appears to be concentrated on products that are the fastest (and to a lesser extent the second fastest) in the industry, with no effect for other products. This is not driven by the possibility that competition has an effect when there are relatively few firms offering a product, but has no marginal effect when the number of competitors grows beyond some critical number: the average number of firms that offer the fastest and second fastest product in the industry is approximately the same as the number of firms that offer slower products. The finding that competition has a stronger effect on prices for fast products, combined with the higher prices these products command, suggests that brand differentiation is stronger at the top of the market, while consumers perceive products at the bottom of the market as fungible across firms. Thus, an increase in the number of brands that offer a top of the line product reduces the pricing power of a firm that offers such a product, by increasing the probability that "top-of-the-line consumers" find some of these additional brands as being close substitute to this firm's product. By contrast, for low ranked products, an increase in the number of brands has no effect as these products are already considered (by the consumers likely to purchase them) to be close substitutes to each other.

In Panels $\mathrm{B}$ and $\mathrm{C}$ of Table 3, we report the price effects of two product entry simulations that combine multiple effects. Though many possible simulations of product entry are possible we focus on two different types of entry of top-of-the-line products. In the first simulation (Panel B), we consider a firm that is currently offering the 2 nd and 3rd fastest products in the industry and adds to its line-up the fastest product in the industry. We compute the effect that this introduction has on the two products that were hitherto its highest speed offerings. We assume that the firm has dropped one of its low speed product offerings and thus that the total number of products that it offers is the same. Industry offerings are held constant. The price effect of the introduction on the price of what was hitherto the fastest product of the firm is the composite of two effects. The first effect is that the previously 2nd fastest chip in the industry and the fastest chip of the firm has now become the 2nd fastest chip in the industry and the 2nd fastest chip of the firm; this tends to push 
down the price of the computer by $3.43 \%$. The second effect is that a chip with the next higher clock speed was hitherto not offered, but now is; this tends to push up the price of this computer by $1.41 \%$, leading to a net change of $-2.02 \%$. The price effect in what was hitherto the 2 nd fastest computer of that firm has only one component. It is the effect of that computer becoming the 3rd fastest in both the firm and the industry from being the 2nd fastest in the firm and 3rd fastest in the industry, and equal to a price decline of $2.29 \%$.

In the second simulation (Panel C), we consider the introduction of a new chip by Intel, which is not adopted by a firm (though it is adopted by other firms). We report the effect of this introduction to the price of the product offerings of the non-adopting firm, under the assumption that the number of competing products of the same clock speed has not changed, and is equal to 6 (the nearest integer to the average value of competing chips in the sample). The price effect on that firm's fastest chip has two components. The first component is that a computer that was the fastest in a firm's product line and also the fastest available in the industry is now only the second fastest available in the industry (though it continues to be the fastest in the firm's product line). This component leads to a price reduction of $9.73 \%$. The second component involves the change in prices due to the smaller coefficient on the number of competing firms for the 2 nd fastest products relative to the value of the coefficient for the faster products. This partially mitigates the price decrease, and leads to a net effect of a price decline of $6.85 \%$. The price effect of the new chip introduction on the firm's second fastest product offering is also characterized by these two opposing effects, leading to a net reduction in price of $0.91 \%$. Finally, the effect of the new product introduction on the firm's third fastest product has only the first component and insignificant in magnitude (and in fact of positive sign). This likely reflects the fact that personal computers that are far from the industry frontier are probably very competitively priced, and do not compete with the top-of-the-line models, either directly or indirectly though the pricing of intermediate products.

The results show that even in a market where product substitutability is high, firms are able to price discriminate on the basis of the relative performance of their own product offerings. The results also provide an indication that simple models of pure vertical differentiation are insufficient 
to capture observed pricing behavior, as they predict no systematic relationship between the pricing of a product and its relative location on a firm line-up (after controlling for product characteristics). Rather, to capture the presence of higher mark-ups toward the top of the product line and the weaker competition of firms at that level, one would likely need to adopt models that allow for stronger brand preferences among consumers with higher willingness to pay for premium products.

\section{References}

Ariely, D., G. Loewenstein, and D. Prelec [2003] "Coherent Arbitrariness: Stable Demand Curves Without Stable Preferences," Quarterly Journal of Economics, 118, 73-105.

Barron, J.M., Taylor, B.A. and J.R. Umbeck [2000] "A Theory of Quality-Related Differences in Retail Margins: Why There is a 'Premium' on Premium Gasoline," Economic Inquiry, 38, 550-569. Deltas, G. and E. Zacharias [2004] "Sampling Frequency and the Comparison Between Conformable Matched Model and Hedonic Regression Price Indexes," Journal of Business and Economics Statistics, 22, 94-106.

Deltas, G. and E. Zacharias [2006] "Entry Order and Pricing Over the Product Cycle: The Transition from the 486 to the Pentium Processor, International Journal of Industrial Organization, 24, 1041-1069.

Gabszewicz, J.J. and J.F. Thisse [1979] "Price Competition, Quality and Income Disparities," Journal of Economic Theory, 20, 340-359.

Gilbert, R.J. and C. Matutes [1993] "Product Line Rivalry with Brand Differentiation," Journal of Industrial Economics, 41, 223-240.

Itoh, M. [1983] "Monopoly, Product Differentiation and Economic Welfare," Journal of Economic Theory, 31, 88-104.

Katz, M. [1984] "Firm Specific Differentiation and Competition among Multiproduct Firms," Journal of Business, 56, S149-S166.

Manez Castillejo, J. A. [1999] "Price Competition and Price Dispersion in the UK Supermarkets," working paper, Departamento de Economa Aplicada II Universitat de Valencia.

Mussa, M. and S. Rosen [1978] "Monopoly and Product Quality," Journal of Economic Theory, $18,301-317$.

Shaked, A. and J. Sutton [1982] "Relaxing Price Competition Through Product Differentiation," 
Review of Economic Studies, 49, 3-13.

Siebert, R. [2003] "The Introduction of New Product Qualities by Incumbent Firms: Market Proliferation versus Cannibalization," Discussion Paper SP II 2003-11, Wissenchaftszentrum Berlin. Stengos, T. and L. Zacharias [2006] "Intertemporal Pricing and Price Discrimination: A Semiparametric Hedonic Analysis of the Personal Computer Market," Journal of Applied Econometrics, 21, 371-386.

Tirole, J. The Theory of Industrial Organization, MIT Press, Cambridge MA, 1988.

Verboven, F. [1999] "Product Line Rivalry and Market Segmentation, with an Application to Automobile Optional Engine Pricing," Journal of Industrial Economics, 47, 399-425.

Verboven, F. [2002] "Quality-based Price Discrimination and Tax Incidence: Evidence from Gasoline and Diesel Cars," RAND Journal of Economics, 33, 275-297.

White, H. [1980] "A Heteroskedasticity-Consistent Covariance Matrix Estimator and a Direct Test for Heteroskedasticity," Econometrica, 48, 817-838.

Wooldridge, J. Econometric Analysis of Cross Section and Panel Data, MIT Press, Cambridge MA, 2002.

Yang, H. and L. Ye "Nonlinear Pricing, Market Coverage, and Competition," Theoretical Economics, 3, 123-153.

Zhang, W., Lee, S.-Y., and X. Song [2002] "Local Polynomial Fitting in Semivarying Coefficient Model," Journal of Multivariate Analysis, 82, 166-188. 
Table 1. Descriptive Statistics of Pruduct Line and Competition Variables.

Binary Variables on Relative Product Position

No. of Observations \% of Observations Average Price

\begin{tabular}{lrrl}
\hline \hline Firm1Industry1 & 1,151 & $12.32 \%$ & $\$ 3,339$ \\
Firm1Industry2 & 338 & $3.62 \%$ & $\$ 3,306$ \\
Firm1Industry3 & 59 & $0.63 \%$ & $\$ 3,302$ \\
Firm1Industry4+ & 37 & $0.40 \%$ & $\$ 3,001$ \\
Firm2Industry2 & 1,311 & $14.04 \%$ & $\$ 2,944$ \\
Firm2Industry3 & 193 & $2.07 \%$ & $\$ 2,571$ \\
Firm2Industry4+ & 128 & $1.37 \%$ & $\$ 2,693$ \\
Firm3Industry3 & 1,238 & $13.26 \%$ & $\$ 2,512$ \\
Firm3Industry4+ & 651 & $6.97 \%$ & $\$ 2,339$ \\
Firm4+ & 4,233 & $45.33 \%$ & $\$ 2,111$ \\
Other Binary Variables & & & \\
Above & 6,482 & $69.41 \%$ & $\$ 2,395$ \\
Below & 6,520 & $69.81 \%$ & $\$ 2,646$ \\
\hline Selected Other Variables & Mean & Std. Deviation & \\
Price & $\$ 2,520$ & $\$ 770$ & \\
NumOfSameChip & 5.89 & 2.29 & \\
NumOfSameChip1 & 0.70 & 1.98 & \\
NumOfSameChip2 & 1.05 & 2.36 & \\
FirmModels & 6.18 & 1.81 & \\
TimeOfferedByFirm & 10.53 & 7.40 & \\
\hline \hline
\end{tabular}

Notes: $\mathrm{N}=9339$. Summary statistics of product characteristics (including brand) and time dummies are ommitted. See Deltas and Zacharias $(2004,2006)$ for information on these variables, and the text for further details. 
Table 2. Estimation Results.

\begin{tabular}{|c|c|c|c|c|c|}
\hline Variable & $\begin{array}{c}\text { no time } \\
\text { interactions }^{(\mathrm{a})}\end{array}$ & $\begin{array}{l}\text { quadratic time } \\
\text { interactions }^{(b)}\end{array}$ & $\begin{array}{l}\text { semi-parametric } \\
(\mathrm{L}=2, \mathrm{~h}=10)^{(\mathrm{c})}\end{array}$ & $\begin{array}{c}\text { quadratic time } \\
\text { interactions, cross- } \\
\text { section } \\
\text { identification }^{(\mathrm{b})} \\
\end{array}$ & $\begin{array}{c}\text { quadratic time } \\
\text { interactions - } \\
\text { linear model }^{(\mathrm{d})} \\
\end{array}$ \\
\hline \multirow{2}{*}{ Firm1 Industry $1^{(\mathrm{e})}$} & 0.1687 & 0.1632 & 0.1583 & 0.2887 & 532.0 \\
\hline & 0.0254 & 0.0233 & 0.0250 & 0.1127 & 65.4 \\
\hline \multirow{2}{*}{ Firm1 Industry2 } & 0.1365 & 0.1102 & 0.1010 & 0.0974 & 342.3 \\
\hline & 0.0237 & 0.0205 & 0.0228 & 0.0280 & 56.7 \\
\hline \multirow{2}{*}{ Firm1 Industry3 } & 0.1008 & 0.0879 & 0.0851 & 0.0846 & 291.7 \\
\hline & 0.0255 & 0.0170 & 0.0224 & 0.0188 & 52.4 \\
\hline \multirow{2}{*}{ Firm1 Industry4+ } & 0.0697 & 0.0656 & 0.0619 & 0.0620 & 289.1 \\
\hline & 0.0274 & 0.0223 & 0.1447 & 0.0221 & 57.9 \\
\hline \multirow{2}{*}{ Firm2Industry2 $2^{(\mathrm{e})}$} & 0.1097 & 0.0746 & 0.0698 & 0.0665 & 261.5 \\
\hline & 0.0165 & 0.0158 & 0.0177 & 0.0241 & 40.7 \\
\hline \multirow{2}{*}{ Firm2Industry3 } & 0.0664 & 0.0445 & 0.0395 & 0.0365 & 127.4 \\
\hline & 0.0108 & 0.0102 & 0.0125 & 0.0424 & 25.4 \\
\hline \multirow{2}{*}{ Firm2Industry4+ } & 0.0558 & 0.0495 & 0.0471 & 0.0424 & 140.5 \\
\hline & 0.0188 & 0.0150 & 0.0152 & 0.0151 & 39.5 \\
\hline \multirow{2}{*}{ Firm3Industry3 $3^{(\mathrm{e})}$} & 0.0497 & 0.0193 & $\underline{0.0141}$ & 0.0156 & 70.1 \\
\hline & 0.0077 & 0.0071 & 0.0083 & 0.0096 & 15.5 \\
\hline \multirow{2}{*}{ Firm3Industry4+ } & 0.0344 & 0.0304 & 0.0281 & 0.0271 & 60.2 \\
\hline & 0.0088 & 0.0076 & 0.0084 & 0.0076 & 18.7 \\
\hline \multirow{2}{*}{ NumOfSameChip } & -0.0004 & 0.0008 & 0.0011 & 0.0003 & 2.5 \\
\hline & 0.0011 & 0.0009 & 0.0011 & 0.0009 & 2.0 \\
\hline \multirow{2}{*}{ NumOfSameChip1 } & -0.0048 & -0.0116 & -0.0118 & -0.0089 & -40.3 \\
\hline & 0.0030 & 0.0027 & 0.0030 & 0.0041 & 8.1 \\
\hline \multirow{2}{*}{ NumOfSameChip2 } & -0.0032 & -0.0047 & $\underline{-0.0044}$ & -0.0053 & -17.4 \\
\hline & 0.0026 & 0.0021 & 0.0025 & 0.0034 & 5.9 \\
\hline \multirow{2}{*}{ FirmModels } & -0.0021 & -0.0026 & -0.0021 & -0.0031 & -5.0 \\
\hline & 0.0025 & 0.0023 & 0.0025 & 0.0023 & 6.0 \\
\hline \multirow{2}{*}{ Above } & $\underline{0.0149}$ & 0.0138 & 0.0121 & 0.0152 & 22.0 \\
\hline & 0.0083 & 0.0068 & 0.0083 & 0.0065 & 16.2 \\
\hline \multirow{2}{*}{ Below } & 0.0226 & 0.0222 & 0.0195 & 0.0172 & 52.5 \\
\hline & 0.0055 & 0.0044 & 0.0056 & 0.0046 & 10.6 \\
\hline \multirow{2}{*}{ TimeOfferedByFirm } & -0.0009 & -0.0019 & -0.0022 & -0.0019 & $\underline{-2.0}$ \\
\hline & 0.0005 & 0.0005 & 0.0006 & 0.0005 & 1.1 \\
\hline R-squared & 0.8989 & 0.9273 & 0.9297 & 0.9280 & 0.9467 \\
\hline
\end{tabular}

Notes: (a) Non-reported variables: In(hard-drive), In(RAM), In(speed), Pentium dummy, In(speed) * Pentium, multimedia dummy, CD-ROM dummy, 15 inch monitor dummy, 17 inch monitor dummy, brand dummies, brand dummies * Pentium, time dummies, time dummies * Pentium. (b) Non-reported variables: As in 1st model, plus all non-reported variables (except for time dummies) are interacted with the trend and trend-squared. (c) See text on non-reported variables and estimation details. (d) Non-reported variables: As in 2nd model, but not in logs. (e) For 4th model, these parameters are the average of interactions with quarterly dummies. For all specifications, $N=9,339$. Robust standard errors, clustered at the firm-chip-month level, are reported in italics below the parameter estimates. Semi-parametric regression standard errors based on a block bootstrap. Bold parameters indicate significance at $5 \%$ level, underlined parameters indicate significance at $10 \%$ level. See text for details. 
Table 3. Product Pricing and Product Position Relative to Firm and Industry Offerings.

Panel A: Average of log-linear specifications

Position in Position in Industry-wide Offerings

\begin{tabular}{lrrrr} 
Firm Offerings & Fastest chip & 2nd fastest chip & 3rd fastest chip & 4th or slower chip \\
\hline \hline Fastest chip & $21.50 \%$ & $11.77 \%$ & $9.37 \%$ & $6.69 \%$ \\
2nd fastest chip & & $8.34 \%$ & $4.78 \%$ & $4.99 \%$ \\
3rd fastest chip & & $2.50 \%$ & $3.04 \%$ \\
\hline \hline
\end{tabular}

Panel B: Effects of the introduction of the top-of-the line chip by a firm on its existing products:

Position in Industry-wide Offerings

Fastest chip 2nd fastest chip 3rd fastest chip 4th or slower chip

\begin{tabular}{llll}
\hline \hline Mean of log-linear models & $-2.02 \%$ & $-2.29 \%$ & not applicable \\
\hline \hline
\end{tabular}

Panel C: Effects of the introduction of a new top-of-the line chip in the industry on a non-adopter:

Position in Industry-wide Offerings PRIOR to the indroduction of the new chip

\begin{tabular}{lcccc} 
& Fastest chip & 2nd fastest chip & 3rd fastest chip & 4th or slower chip \\
\hline \hline Mean of log-linear models & $-6.85 \%$ & $-0.91 \%$ & $0.55 \%$ & not applicable \\
\hline \hline
\end{tabular}

Notes: The percentage effect in the log-linear specification is based on exp(average coefficient)-1. Panel A: Prices are relative to product offerings that are the fourth or lower in a firm's product line-up in terms of computer speed. The values of all other firm-level variables is held constant in the comparisons, i.e., that the pattern of gaps in a firm's offerings below its fastest model is kept constant. Panel B: Figures take into account nearest-neigbhor price effects. Firm is assumed to drop one low speed product. Panel C: Figures take into account the change in the industry competition. See text for details. 